\title{
MRI Findings in Pott's Spine with its Histopathological Correlation
}

\author{
Sangeeta Saxena ${ }^{1}$, Bhajan lal ${ }^{2}$, Dharm Raj Meena ${ }^{3}$, Harsh Vardhan ${ }^{4}$, Abhishek Gupta ${ }^{2}$, Chandraveer Singh Godara ${ }^{2}$ \\ ${ }^{1}$ Senior professor, Department of Radio-diagnosis, Government Medical College, Kota, Rajasthan, INDIA, ${ }^{2}$ Resident doctor, Department of Radio-diagnosis, \\ Government Medical College, Kota, Rajasthan, INDIA, ${ }^{3}$ Professor \& HOD, Department of Radio-diagnosis, Government Medical College, Kota, Rajasthan, INDIA, \\ ${ }^{4}$ Assistant professor, Department of Radio-diagnosis, Government Medical College, Kota, Rajasthan, INDIA.
}

\section{Abstract}

Background: MRI is now the preferred imaging modality and preferred technique to define the activity and extent of infection for patients with suspected spinal tuberculosis. The objective of the study was to describe diagnostic accuracy, sensitivity and specificity of MRI in diagnosis of spinal tuberculosis and various radiological features of spinal tuberculosis (TB) on magnetic resonance imaging (MRI). Subjects and Methods: Out of total 45 cases of suspected tuberculosis in our study we given 38 case as spinal spondylo-discitis out of which 36 cases accurately diagnosed by MRI proved by histopathology or culture reports. And 7 cases of given non tubercular out of which 1 case came to be tubercular spondylodiscitis on histopathology. MRI case records of these 37 patients with proven tuberculosis retrospectively analysed. Results: The majority of the patients were males 60\% (22/37) and most common age group is 31 to 50 years age group (45\%). The most common clinical presentation was backache $81 \%(n=30)$ followed by fever $22 / 37$ cases $(59.45 \%)$. The Thoraco-lumbar spine was the commonest site of the disease $(38.64 \%)$ followed by the lumbar region $(31.82 \%)$. An intervertebral disc involvement, pre and paravertebral collections, subligamental extension of the abscess were commonly seen, with an epidural collection occurring in more than $75 \%$ of the cases. In addition few cases also showed single vertebral involvement (n-3) and few cases shows skip lesions. Conclusion: The MRI scan is highly sensitive in the detection of various pathological processes of spinal tuberculosis and their pattern of occurrence. The extent of soft tissue involvement disease is best assessed by MRI which help in guiding the surgical treatment as well as to monitor the response to treatment during follow up.

Keywords: Spinal tuberculosis, Magnetic resonance imaging, Epidural Abscess, Antituberculous treatment, Cold abscess, Mycobacterium tuberculosis.

Corresponding Author: Dr. Bhajan lal, Resident doctor, Department of Radio-diagnosis, Government Medical College, Kota, Rajasthan, India.

Received: May 2019

Accepted: June 2019

\section{Introduction}

Spinal tuberculosis is a frequently encountered extra pulmonary form of the disease. In developing country like India there is high burden of tuberculosis. Spinal infections represent $2-4 \%$ of all cases of osteomyelitis and the most common causative organisms are tubercular and represent a serious disease, which requires early diagnosis and prompt treatment. It is caused by the Mycobacterium tuberculosis bacteria, most commonly by a haematogenous spread of the infection via the venous plexus of Batson. ${ }^{[1,2]}$ Spinal tuberculosis show a subacute or chronic course. MRI appearance of tuberculosis mimics various other pathological processes and these can be categorized into infectious and neoplastic lesions. The major differential diagnoses include low grade infections like atypical mycobacteria, brucella and fungal infections and neoplasms like lymphomas, multiple myelomas and metastases. These infections generally involve the intervertebral discs while they are spared in the neoplasms. ${ }^{[3]}$ It is most prevalent in adults with no sex predominance. The worst complications of tuberculosis of the spine are para or quadriplegia, hemiplegia or monoplegia, with an incidence of paraparesis which varies from $27-47 \%$. ${ }^{[3]}$ Tuberculosis is the most common cause of non-traumatic paraplegia in the most parts of world. ${ }^{[3,4]}$ The neurological symptoms tend to occur in the acute as well as the late stages of the disease. The neurological symptoms include radicular pain, the severe cauda equina syndrome and spinal cord compression.

The thoraco-lumber segments are the preferred sites in which infection spreads, followed by the lumbar levels. The disease often involves multiple contiguous vertebral bodies with the intervening disk, but two vertebral involvement with the intervening disc is also common and characterizes the disease. It remains unclear whether the multivertebral disease is a consequence of hematogenous dissemination or it is a direct subligamentous, paraspinal or subarachnoid spread disease, although subligamentous spread seems most likely. The infection typically begins from the anterior part of vertebral body, spreads to the disc and causes bone destruction and formation of abscess. Subligamental extension of abscess beneath the anterior longitudinal ligament and the intervertebral disc is involved with subsequent loss in disc height. As the vertebral bodies collapse into each other, a sharp angulation (or kyphos) 
develops

This review focus on to describe the MRI spectrum of findings in patients with spinal tuberculosis and to demonstrate, analyze, and evaluate Magnetic Resonance Imaging as a valuable non invasive diagnostic tool in spinal tuberculosis.

\section{Subjects and Methods}

The study included a total of 45 cases of suspected spinal tuberculosis on MRI. The patients who were referred to the department of radiology from the orthopaedics, pulmonary medicine, General Medicine, Paediatric Departments on the basis of their clinical prescntation. A detailcd history along with complete clinical examination and laboratory investigations will be done before the MRI examination. Before evaluating a patient by MRI imaging informed consent will be obtained from the patient or guardian. Study Period is one year from september 2017 to august 2018. It is prospective study design, Descriptive and analytical study.

The data obtained was compiled systematically, transformed from a pre-coded pro-forma to a computer and a master table was prepared. The total data was distributed meaningfully and presented as individual tables along with graphs. Descriptive and Inferential statistical analysis has been carried out in the present study using computer software (SPSS Trial version 23 and primer). The qualitative data were expressed in proportion and percentages, and the quantitative data expressed as mean and standard deviations. The difference in proportion was analyzed by using chi square test. Significance level for tests were determined as $95 \%(\mathrm{P}<0.05)$. The diagnostic accuracy, sensitivity, and specificity of the various imaging modalities were calculated. Significance level for tests were determined as $95 \%(\mathrm{P}<0.05)$.

\section{The following features were assessed by MRI: \\ 1. Spondilitis or hyperintense vertebral body \\ 2. Discitis or T2 hyperintense intervertebral disc \\ 3. Cord oedema \\ 4. Epidural collection or abscess \\ 5. Cord compression or spinal canal narrowing \\ 6. Which segment of spine involved \\ 7. Collection in psoas muscle or paraspinal collection \\ 8. Vertebral body abscess \\ 9. Histopathological correlation \\ 10. Subligamentous spread \\ 11. Number of vertebral segments affected}

\section{Results}

Out of total 45 cases of suspected tuberculosis in our study we given 38 case as tubercular spondylo-discitis out of which 36 cases accurately diagnosed by MRI proved by histopathology or culture reports. And 7 cases of given non-tubercular out of which 1 case came to be tubercular spondylodiscitis on histopathology. MRI case records of these 37 patients with proven tuberculosis retrospectively analysed.

Of the 37 patients with spinal tuberculosis in our study more than half were males $(60 \%)$ and the majority (45\%) of these were in the 31 to 50 year age group. The most common clinical presentation was backache $81 \%(n=30)$ followed by fever $22 / 37$ cases (59.45\%), neurological complication radiculopathy and Para-paresis in (13/37) $35.2 \%$ cases and history of previous or current tuberculosis in $(12 / 37) 32.4 \%$ cases.

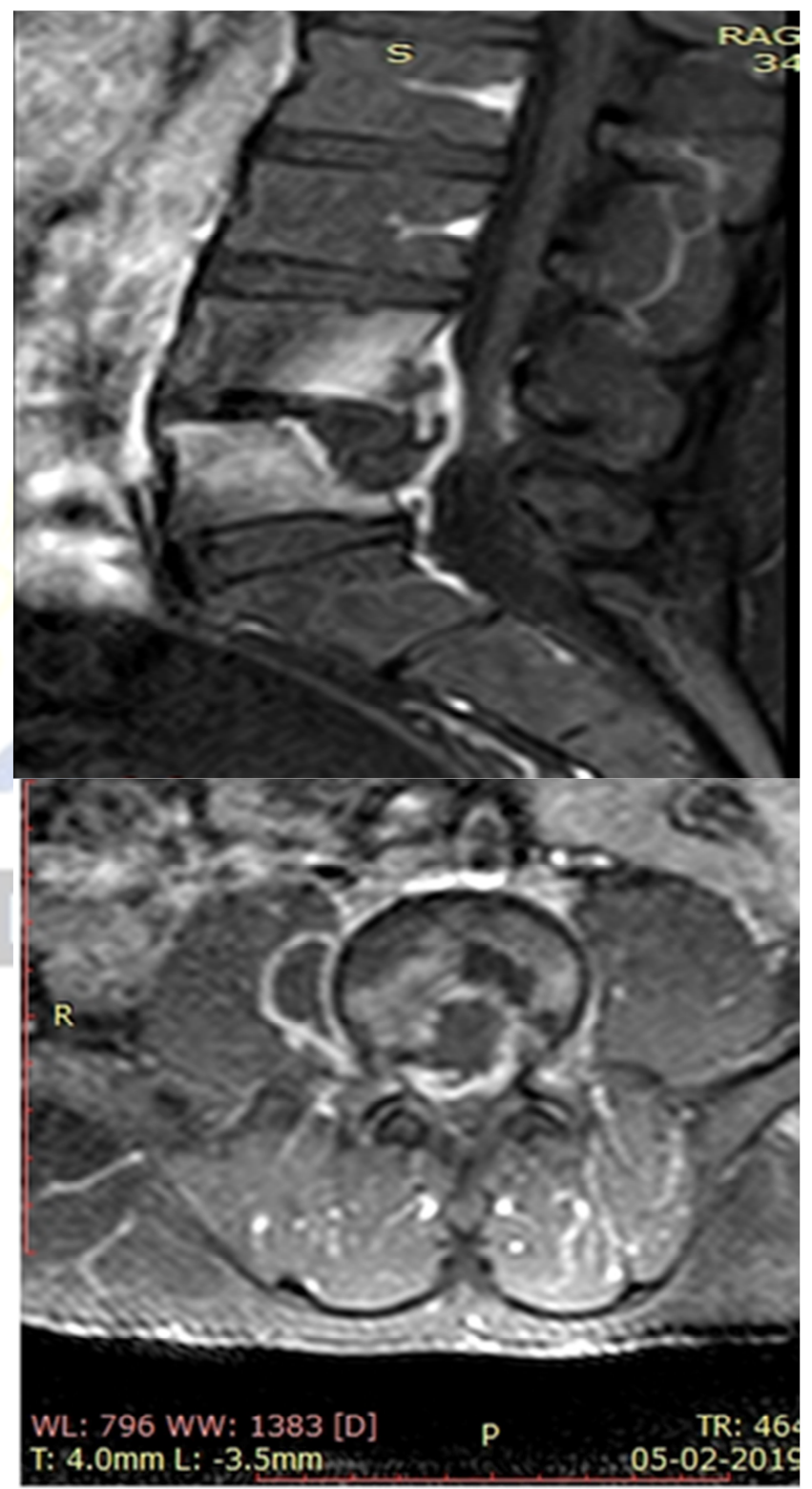

Figure 1: Saggital fat supressed $\mathrm{T} 1 \mathrm{c}+(\mathrm{A}) \&$ axial T1c+ (B) heterogenous enhancement of $\mathrm{L} 4$ and $\mathrm{L} 5$ vertebral body and small epidural collection and end plate destruction. Small amount of paraspinal collection ( short arrow )also noted. Vertebral body abscess with smooth enhancing wall(long void arrow).

An intervertebral disc involvement was a common finding which was noted in (35/37) $94.5 \%$ tubercular spondylodiscitis cases and (7/8) $87.5 \%$ of non-tubercular 
cases. Two vertebra involvement noted $34 \%$ of cases and more than two vertebra involvement is present in $69 \%$ of cases. Single vertebral involvement also present in few cases (2/37) $5.4 \%$ of cases. Most of non tubercular spondylodiscitis cases present with 2 vertebral involvement (7/8) $87.5 \%$ of cases. Multiple vertebral involvements present as most commonly as Multi-level non-contiguous involvement however few cases of skip lesion also present in our study. Skip lesion is present in (2/37) 5.4\% of cases. The thoracolumbar spine involvement in was the commonest site of the tubercular disease (15/37) $40.5 \%$ of cases, followed by disease in the lumbar spine. However non tubercular spondylodiscitis present majority in lumbar spine $(5 / 8) 62.5 \%$ of cases. Para spinal collection present in (31/37) 83.7\% tubercular cases. However paraspinal collection is present in minority of cases of non-tubercular spondylodiscitis (1/8) $12.5 \%$ of cases.

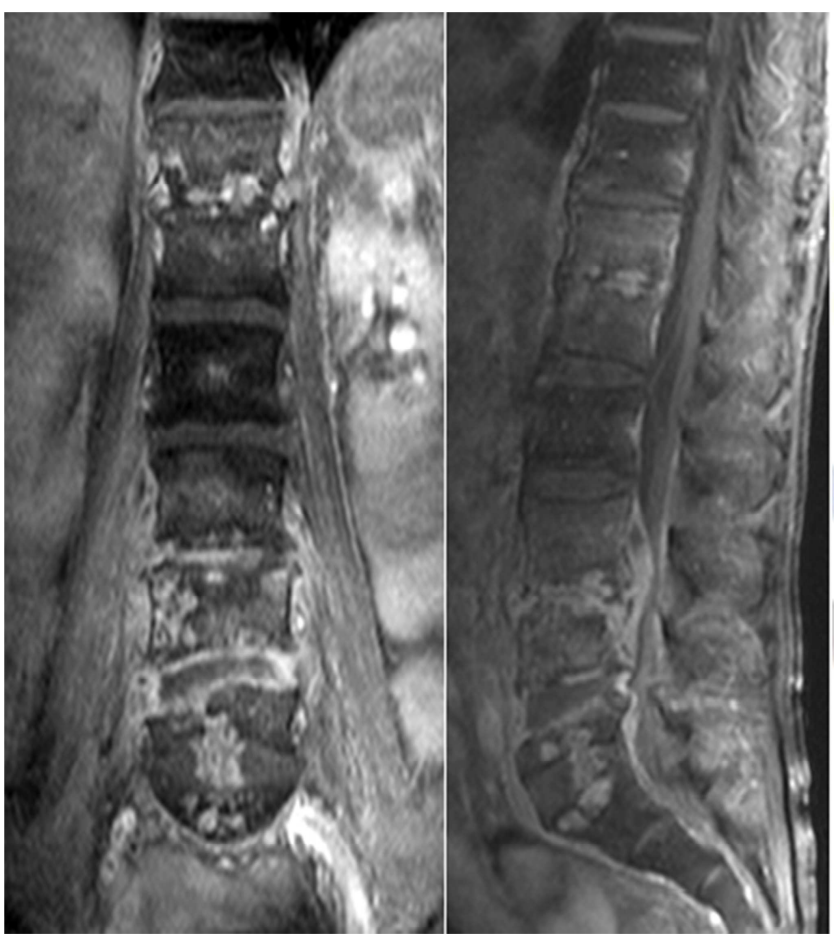

Figure 2: Coronal contrast enhanced fat supressed T1 weighted image shows atypical skip segment involvement of tubercular spondylodiscitis. Figure 2a:- Spondylitis changes noted in L2, L3, L4 and L5 vertebral body and intervening disc with inflammatory changes and small collection also noted in paraspinal muscles. Spondylodiscitis changes noted in T11T12 vertebral level. Intra vertebral abscess formation also noted in L5 vertebral body. Figure 2b:- sagittal contrast enhanced fat supressed $T 1$ weighted image shows spodylodiscitis changes in L2 to L5 and T11-T12 level(black arrow).

The paraspinal collection in non-tubercular cases is small in size with irregular wall. Majority of collection in tubercular cases are large in size with well-defined thin enhancing wall in tubercular spine involvement. Intraosseous vertebral body abscess present in $12(32.5 \%)$ tubercular cases however it is present in nontubercular cases also (2/8) $25 \%$ of cases. Sub ligamentous spread present in was observed in
(20/37) $54 \%$ of cases. No sub ligamentous spread of infection noted in non-tubercular spondylodiscitis cases. Sub ligamentous spread commonly associated with multiple vertebral segment involvement. Epidural collection is frequent and important finding of spinal tuberculosis and present in (28/37) $75 \%$ of cases. Epidural collection commonly causing spinal canal stenosis and neurological symptoms. Epidural collection is present in (13/37) $35.1 \%$ of cases of non tubercular spondylitis cases. Complete vertebral body destruction is also commonly present in tubercular spine involvement.

Overall MRI showed a sensitivity of $97.2 \%$, a specificity of $87.5 \%$ and an accuracy of $94.7 \%$ in diagnosing TB when compared to pyogenic infection. Their most indicative signs of vertebral TB were: well-defined Para spinal abnormal signal, thin and smooth abscess wall, combination of both findings, presence of soft tissue or intraosseous abscess, sub ligamentous spread to three or more vertebral levels, involvement of multiple vertebral bodies, para-discal pattern of involvement, thoracolumbar spine localization and hyper intense signal on T2-weighted images.

\section{Discussion}

Tuberculous spondylitis is defined as an infection by mycobacterium tuberculosis involving one or more components of the spine, namely the vertebra, intervertebral discs, Para spinal soft tissues and the epidural space. It is one of the most common manifestations of musculoskeletal TB.

The mean age of Histo-pathological confirmed diagnosis of TB cases was $41.5 \pm 18$ years and most common age group is 31 to 50 years of age. Sinan et al. (2004). ${ }^{[6]}$ Found $43 \%$ cases in their study in the age group of 30-49 years. Khalequzzaman S1 et al. ${ }^{[8]}$ (2012) study, the most common age presenting with tuberculous spinal infection is between 31 to 40 years $(43 \%)$, Shashikumar MR et al. $2015,{ }^{[7]}$ The study included total 40 patients, with an age range of 21-60 years with majority of them in the $31-40$ year age group $(50 \%)$.

According to clinical features there were $81 \%(n=30)$ out of 37 cases of positive parameter where back pain was found positive Fever were present in 22/37 cases (59.45\%). Sinan et al (2004), ${ }^{[6]}$ The most common clinical presentation was backache $(73.3 \%)$ followed by fever $(63.3 \%)$ and malaise (36.6\%). Shashikumar MR et al. ${ }^{[7]} 2015$ The most common clinical presentation was backache $(77 \%)$ with a localized kyphotic deformity followed by fever $(62.5 \%)$, malaise (47.5\%) and weight loss $(22.5 \%)$.

DISCITIS was observed in $94.5 \%$ in our study. Sinan et al. (2004) disc destruction (osteitis and discitis) in 10 cases $(10 / 11=90 / 91 \%)$. Discitis has no significant association with spinal tuberculosis $(p=0.69)$ In our study two vertebra involvement noted $15(34 \%)$ of cases and more than two vertebra involvement is present in $26(69 \%)$ of cases $(p=0.004)$. Single vertebral body involvement found only $6.82 \%$ of cases in our study. Khalequzzaman S1 et al. ${ }^{[8]}$ (2012) single vertebral body involvement found only 7\% of cases. Lolge et al. ${ }^{[9]}$ showed fewer cases $(1.69 \%)$ of solitary 
vertebral body involvement. Jain, ${ }^{[10]}$ suggested that multiple level tubercular lesions in the spine were observed in 16.3$71.4 \%$ of the cases. Multiple vertebral segment involvement is higher than most western studies due to advanced disease at presentation and the route of transmission due to hematogeneous and subligamentous spread. Kaila Rajiv et al retrospectively reviewed whole spine MRI of all cases over 3 years to identify the incidence of multiple level noncontiguous vertebral tuberculosis and found it to be $71.4 \%$. and concluded that this non contiguous vertebral involvement is more frequent than previously thought.

Sub ligamentous spread present in was observed in (20/37) $54 \%$ of cases in our study $(p=0.01)$. Garcia et al, ${ }^{[11]}(2013)$ Subligamentous spread was present in $40 \%$ of cases with associated multilevel infections. Garcia et al, ${ }^{[11]}$ (2013) Subligamentous spread was present in $40 \%$ of cases with multilevel infections $(n=8)$. In our study, paraspinal collection was observed 31/37 (83.7\%). Statistically significant association of Paraspinal collection with histopathological diagnosis was observed $(\mathrm{p}=0.001)$. Osborn et $\mathrm{al}^{[12]}$ stated that a paraspinal abscess was present in $55-95 \%$ of the cases. Chang Ming-Chau et al., ${ }^{[13]}$ and Gehlot PS et al., ${ }^{[14]}$ emphasized the advantage of MRI were MRI could detect paravertebral component in $82 \%$ and $80 \%$ patients respectively. Vertebral body abscess were present $29.72 \%$ $(11 / 37)$. Vertebral body abscess is also present in nontubercular cases $12.5 \%(1 / 8)$. Vertebral abscess shows no Statistically significant association with histopathological diagnosis (0.34). Park et $\mathrm{al}^{[15]}$ (2011) There were no significant differences in the following MR imaging findings paraspinal abscess or intraosseous abscess $(100 \%$ in tuberculous vs $71 \%$ in pyogenic, $\mathrm{p}=0.146$ ).

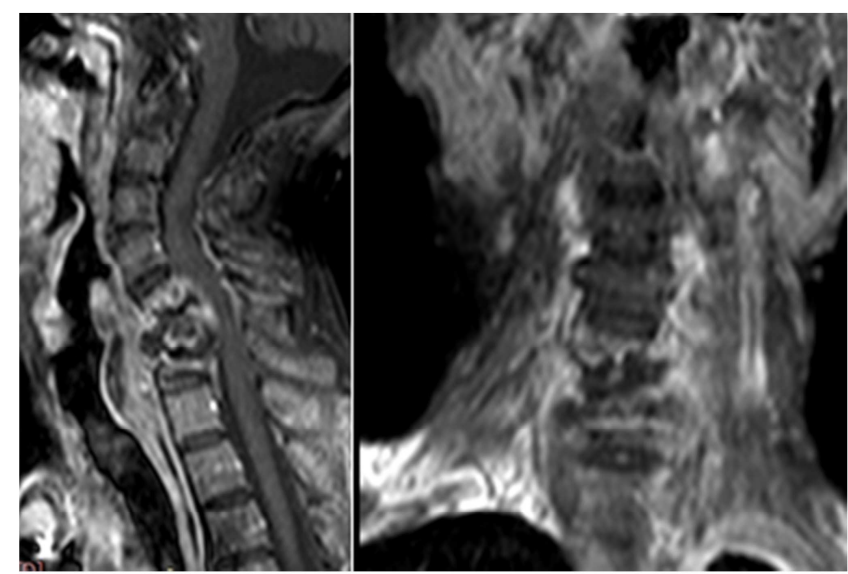

Figure 3: A spondylodiscitis of $\mathrm{C} 6, \mathrm{C} 7$ and $\mathrm{T} 1$ vertebra with focal gibbus deformity and collapse and destruction of vertebra. A paraspinal inflammatory changes also noted.

In our study, sensitivity, specificity, PPV, NPV and diagnostic accuracy of MRI against the histopathological diagnosis $97.2 \%, 87.5 \%, 94.7 \%, 90 \%$ and $94.5 \%$. NaYoung et al. ${ }^{[16]}$ found that MRI showed a sensitivity of 100 $\%$, a specificity of $80 \%$ and an accuracy of $90 \%$ in diagnosing TB when compared to pyogenic infection. Modic et al (1985), ${ }^{[17]}$ MR had a sensitivity of $96 \%$, specificity of $92 \%$, and accuracy of $94 \%$. Our study also shows sensitivity, specificity and diagnostic accuracy is equilent to previous studies.

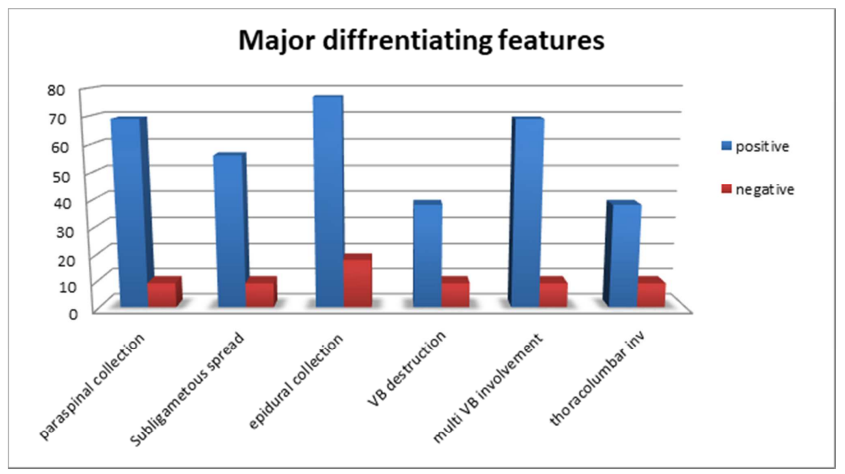

\section{Conclusion}

MRI has excellent sensitivity, specificity and diagnostic accuracy in diagnosis of spinal tuberculosis. Para spinal collection, epidural collection, more than two vertebra involvement, sub ligamentous spread, vertebral body destruction are most important finding for diagnosis of spinal tuberculosis. Incidence of non contiguous vertebral involvement is more frequent than previously thought in this study due to probably due to advanced disease in our cases of indian population and the route of transmission due to hematogeneous and subligamentous spread.

\section{References}

1. Stark DD, Bradley WG. Magnetic Resonance Imaging. 3rd ed. Mosby;. Chapter 86, Thoracic spine 1999; 1851-82.

. Burrill J, Williams CJ, Bain G, Conder G, Hine AL, Misra RR Tuberculosis: A Radiologic Review. Radiographics 2007;27(5):125573. doi: $10.1148 / \mathrm{rg} .275065176$.

3. Garg RK. Tuberculosis of the central nervous system. Postgrad Med J [Internet]. 1999 [cited 20 Feb 2012]:75:133-140 Available from: http://pmj.bmj.com/content/75/881/133.full.

4. Bajwa GR. Evaluation of the role of MRI in spinal Tuberculosis: A study of 60 cases. Pak J Med Sci [Internet]. 2009 [cited 20 Feb 2012];25(6):944-947. Available from: http://www.pjms.com.pk/issues/octdec209/pdf/14.article13.pdf.

5. De Backer AI, Mortelé KJ, Vanschoubroeck IJ, Deeren D, Vanhoenacker FM, et al. Tuberculosis of the spine: CT and MR imaging features. JBR-BTR [Internet]. 2005 [cited 20 Feb 2012];88(2):92-97 Available from: http://www.rbrs.org/dbfiles/journalarticle_0256.pdf.

6. Tariq Sinan, FRCR*; Hana Al-Khawari, FRCR*; Mohammed Ismail, MD+; Abdulmohsen Ben-Nakhi, FRCR*; Mehraj Sheikh, MD, FRCR* Spinal tuberculosis: CT and MRI features .Ann Saudi Med 2004;24(6):437-441

7. Shashikumar MR, Basavaraj SB, Joshi VV, Nanjaraj CP, Rajendrakumar NL. Role of MRI in the evaluation of spinal tuberculosis. Int J Res Med Sci 2015;3(8):1839-43.

8. Khalequzzaman S1, Hoque HW2.Tuberculosis of Spine Magnetic Resonance Imaging (MRI) Evaluation of 42 Cases .medicine today. $2012 ; 24$.

9. Lolge S., Maheshwari M., Shah J., Patkar D., Chawla A. Isolated solitary vertebral body tuberculosis-study of seven cases, Clin Radiol 2003; 58:545-50.

10. Jain A K. Tuberculosis of the spine: A fresh look at an old disease. J Bone Joint Surg Br. 2010 Jul;92-B(70):905-13. doi: 10.1302/0301620X.92B7.24668.

11. Antonio Rivas-Garcia • Silvana Sarria-Estrada $•$ Carme Torrents-Odin - Lourdes Casas-Gomila Imaging findings of Pott's disease • Elisa 


\section{Saxcena et al; MRI Findings in Satt's Spine with its Htistapathalagical Carrelation}

Franquet Eur Spine J (2013) 22 (Suppl 4):S567-S578

12. Osborn AG. Diagnostic Neuroradiology. 1e ed. Elsevier;. Chapter 20, Nonneoplastic disorders of the spine and spinal cord 2009; 820-75.

13. Chang MC, Wu HT, Lee CH, Liu CL et al. Tuberculous spondylitis and pyogenic spondylitis: comparative magnetic resonance imaging feature. Spine. 2006;31(7):782-88

14. Gehlot PS, Chaturvedi S, Kashyap R, Singh V. Pott's spine: retrospective analysis of MRI scan of 70 cases. J Clin Diagn Res. 2012;6(4):1534-38

15. Jong-Han Park,1 Hye-Seon Shin,2 Jong Tae Park,1 Tae Young
Kim,land Ki Seong Eom 1

16. Nam- Hyun Kim, Hwan- MO Lee, Jin- Suck Sub. Magnetic Resonance imaging for the diagnosis of tubercular spondylitis. Spine 1994; 19(21): $2451-2455$

17. Modic MT, Feiglin DH, Piralno DW, Boumphrey F. Weinstein MA Duchesneau PM et al: Vertebral Osteomyelitis: Assessment using MR Radiology. 1985; 158: $157-166$.

Copyright: (C) the author(s), publisher. Asian Journal of Medical Radiological Research is an Official Publication of "Society for Health Care \& Research Development". It is an open-access article distributed under the terms of the Creative Commons Attribution Non-Commercial License, which permits unrestricted non-commercial use, distribution, and reproduction in any medium, provided the original work is properly cited.

How to cite this article: Saxena S, lal B, Meena DR, Vardhan H, Gupta A, Godara CS. MRI Findings in Pott's Spine with its Histopathological Correlation. Asian J. Med. Radiol. Res. 2019;7(1):56-60.

DOI: dx.doi.org/10.21276/ajmrr.2019.7.1.13

Source of Support: Nil, Conflict of Interest: None declared. 